\title{
Performance Evaluation of Low Density Spreading Multiple Access
}

\author{
Mohammed AL-Imari*, Muhammad Ali Imran*, Rahim Tafazolli*, and Dageng Chen ${ }^{\dagger}$ \\ ${ }^{*}$ Centre for Communication Systems Research,University of Surrey, Guildford GU2 7XH, Surrey, U.K. \\ Email: \{m.al-imari, m.imran, r.tafazolli\}@ surrey.ac.uk \\ ${ }^{\dagger}$ Huawei Technologies Co., Ltd. No.2222, Xin JinQiao Road, Shanghai, China, 201206 \\ Email: dagengchen@huawei.com
}

\begin{abstract}
In this paper, we evaluate the performance of Multicarrier-Low Density Spreading Multiple Access (MCLDSMA) as a multiple access technique for mobile communication systems. The MC-LDSMA technique is compared with current multiple access techniques, OFDMA and SC-FDMA. The performance is evaluated in terms of cubic metric, block error rate, spectral efficiency and fairness. The aim is to investigate the expected gains of using MC-LDSMA in the uplink for next generation cellular systems. The simulation results of the link and system-level performance evaluation show that MC-LDSMA has significant performance improvements over SC-FDMA and OFDMA. It is shown that using MC-LDSMA can considerably reduce the required transmission power and increase the spectral efficiency and fairness among the users.
\end{abstract}

Index Terms-Multiple access technique, low density spreading, cubic metric, link-level, spectral efficiency, fairness.

\section{INTRODUCTION}

Next generation mobile communication systems are expected to provide high-date rate services, which requires more advanced and spectral-efficient multiple access techniques. In this regard, multicarrier transmission has been considered as a promising technique for broadband wireless communication [1]. Orthogonal Frequency Division Multiple Access (OFDMA) is one of the major multicarrier transmission techniques that has been adopted as the core technology in existing and upcoming mobile communication standards, such as the 3rd Generation Partnership Project Long Term Evolution (3GPP-LTE) standard [2], and Worldwide Interoperability for Microwave Access (WiMAX) [3]. Single Carrier Frequency Division Multiple Access (SC-FDMA) [4] is a modified form of OFDMA, which has been accepted as an uplink scheme for 3GPP-LTE. SC-FDMA benefits from low envelope fluctuations comparing to OFDMA making it suitable for uplink transmission by user equipment.

A new multiple access concept based on low density spreading has been introduced in [5] to manage the multiuser interference and allow overloaded conditions with affordable multiuser detection complexity. The Low Density Spreading Multiple Access (LDSMA) concept has been extended to multicarrier communication to cope with multipath channel effect. In Multicarrier-LDSMA (MC-LDSMA), the spreading is done with a low density spreading codes with only small number of non-zero chips in each code. Therefore, every data symbol will only be spread on a small subset of subcarriers, and every subcarrier will only be used by a small subset of data symbols that could belong to different users.

In this way, MC-LDSMA technique will be able to exploit the channel and the multiple access interference diversities over the frequency domain. The diversity gain can improve the link-level performance in terms of Block Error Rate (BLER). Furthermore, as there is no exclusivity in the subcarrier allocation like in OFDMA and SC-FDMA, there is plenty of room to exploit the high degree of flexibility of subcarrier allocation. This resource allocation flexibility can utilized by efficient radio resource allocation scheme to enhance the system-level performance such as the spectral efficiency, the number of supported users and fairness among users. Considering these advantages, MC-LDSMA represents a strong candidate for next generation cellular system as a multiple access technique.

In this paper, we will evaluate the performance of $\mathrm{MC}$ LDSMA and compare it with current multiple access techniques; namely SC-FDMA and OFDMA. The aim is to investigate the expected gains of using MC-LDSMA in the uplink for next generation cellular systems. The comparison will be done in Cubic Metric (CM), link-level and systemlevel performance through extensive Monte Carlo simulations. Cubic Metric has been proposed recently as a measure of the envelope fluctuations which can predict the power de-rating more accurately [6], [7]. The main simulation parameters are used to match the 3GPP-LTE standard. It will be shown that MC-LDSMA outperforms OFDMA in CM, link-level and system-level performance. On the other hand, MC-LDSMA has CM higher than SC-FDMA, which is compensated by better link-level performance making MC-LDSMA outperforms SC-FDMA. Also, MC-LDSMA outperforms SC-FDMA in the system-level performance. The results show that using MCLDSMA can significantly improve the system performance in terms of required transmission power, cell coverage, number of supported users in the cell and users' data rates.

This paper is organized as follows: Section II presents the MC-LDSMA system architecture. The CM and link-level performance evaluation are provided in section III. In section IV, we evaluate and compare the system-level performance for the considered multiple access techniques. Finally, section V is devoted to concluding remarks. 


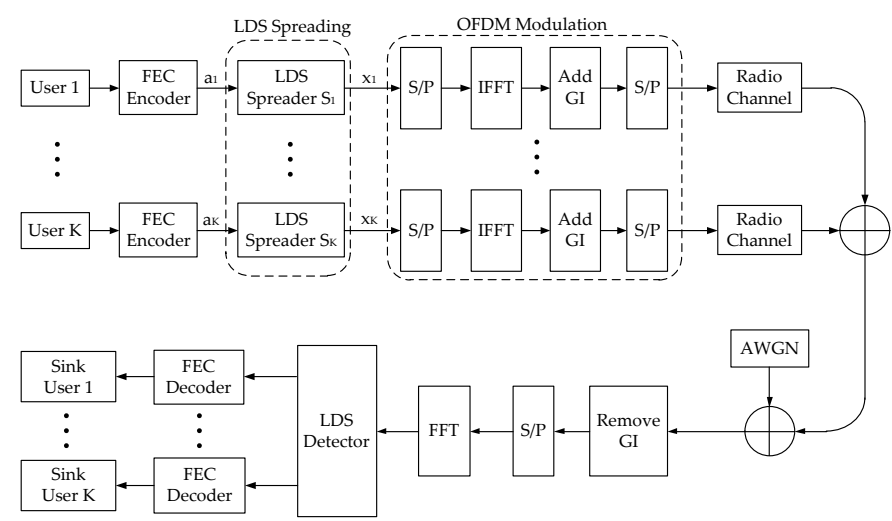

Fig. 1. Uplink MC-LDSMA block diagram.

\section{MC-LDSMA SYSTEM MODEL}

In this section, a single cell uplink MC-LDSMA system model is presented. The conceptual block diagram of an uplink MC-LDSMA system is depicted in Fig. 1. We consider a system with a set of users $\mathcal{K}=\{1, \cdots, K\}$ transmitting to the same base station where the base station and each user are equipped with a single antenna. The total frequency band is divided into a set of subcarriers $\mathcal{N}=\{1, \cdots, N\}$. A user $k \in \mathcal{K}$ can transmit over a subset of the subcarriers, with transmission power $p_{k, n}$ over subcarrier $n \in \mathcal{N}$ subject to individual maximum power constraints $P_{k}: \sum_{n \in \mathcal{N}} p_{k, n} \leq P_{k}$.

Let $\mathbf{a}_{k}$ be a data vector of user $k$ consisting of $M_{k}$ modulated data symbols and denoted as;

$$
\mathbf{a}_{k}=\left[a_{k, 1}, a_{k, 2}, \cdots, a_{k, M_{k}}\right]^{T} .
$$

The signature matrix $\mathbf{S}_{k}$ assigned for the $k$ th user consists of $M_{k}$ Low Density Signatures (LDS);

$$
\mathbf{S}_{k}=\left[\mathbf{s}_{k, 1}, \mathbf{s}_{k, 2} \cdots, \mathbf{s}_{k, M_{k}}\right] .
$$

Where each LDS signature, $\mathbf{s}_{k, m} \in \mathbb{C}^{N \times 1}$, is a sparse vector consisting of $N$ chips. Among these $N$ chips only $d_{v}$ chips have non-zero values, where $d_{v}$ is the effective spreading factor. Each data symbol $a_{k, m}$ will be spread using the $m$ th spreading sequence. Let $\mathbf{x}_{k}=\left[x_{k, 1}, x_{k, 2}, \cdots, x_{k, N}\right]^{T}$ denote the chips vector belongs to user $k$ after the spreading process, which is given by;

$$
\mathbf{x}_{k}=\mathbf{S}_{k} \mathbf{a}_{k} .
$$

So, the whole system's signature matrix has $N$ rows and $M$ columns each containing a unique spreading sequence, where $M$ can be calculated as follows;

$$
M=\sum_{k=1}^{K} M_{k} .
$$

The system overloading, which is the ratio of the number of transmitted symbols to the total number of subcarriers, will be;

$$
\beta=\frac{M}{N} * 100 \%
$$

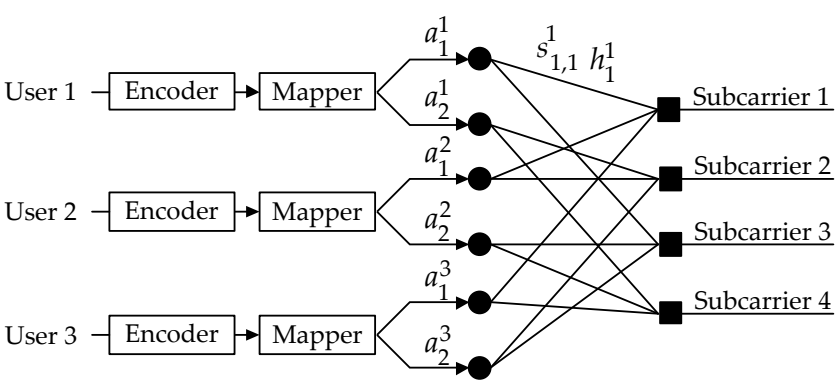

Fig. 2. Graphical representation of MC-LDSMA.

The main advantage of the low density spreading is that the system overloading can be more than $100 \%$ with affordable complexity and close to single user performance. Each user's generated chip will be transmitted over a subcarrier of the OFDM system. Fig. 2 illustrates the MC-LDSMA principle by an example of a system with four subcarriers $(N=4)$, serving three users $(K=3)$ with two data symbols per user ( $M_{1}=M_{2}=M_{3}=2$ ), which means $150 \%$ overloading. Here the effective spreading factor is two $\left(d_{v}=2\right)$ and each three chips sharing one subcarrier $\left(d_{c}=3\right)$, where $d_{c}$ denotes the number of users interfere in each subcarrier. The figure shows in more details the process of low density spreading. As it can be observed that each chip represents a subcarrier of OFDM modulation and the data symbols using the same subcarrier will interfere with each other.

As users are not bound to exclusively use the subcarriers, at the receiver side users' signals that are using the same subcarrier will be superimposed. However, the number of users interfere in each subcarrier is much less than the total number of users, $d_{c} \ll K$. At the receiver side, after performing OFDM demodulation operation, the received signal is given by;

$$
\mathbf{y}=\sum_{k=1}^{K} \mathbf{H}_{k} \mathbf{x}_{k}+\mathbf{v}
$$

where $\mathbf{v}$ is the Additive White Gaussian Noise (AWGN) and $\mathbf{H}_{k}$ is the frequency domain channel transfer function of user $k$

$$
\mathbf{H}_{k}=\operatorname{diag}\left(h_{k, 1}, h_{k, 2}, \cdots, h_{k, N}\right),
$$

where $h_{k, n}$ is the channel gain of user $k$ on subcarrier $n$. This signal $\mathbf{y}$ is passed to LDS multiuser detector (MUD) to separate users' symbols. The LDS structure can be captured by a low density graph, thus the detection of MC-LDSMA can be done using close to optimum chip-level iterated MUD based on Message Passing Algorithm (MPA) presented in [8]. The complexity of the multiuser detection for MC-LDSMA will turn out to be $\mathcal{O}\left(|\mathbb{X}|^{d_{c}}\right)$, which is significantly reduced comparing to complexity of order $\mathcal{O}\left(|\mathbb{X}|^{K}\right)$ for optimal multiuser detection, where $\mathbb{X}$ denotes the constellation alphabet. More details regarding the LDS receiver can be found in [8]. 
TABLE I

CM Simulation PARAMETERS.

\begin{tabular}{|c||c|}
\hline \multicolumn{1}{|c|}{ Parameter } & Value \\
\hline \hline FFT size & 256 \\
\hline Subcarrier spacing & $15 \mathrm{KHz}$ \\
\hline Subcarriers per RB & 12 \\
\hline Number of data RB & $N_{R B}=15$ \\
\hline Effective spreading for MC-LDSMA & $d_{v}=3$ \\
\hline RB per user (MC-LDSMA) & $M_{R B}=3,6,9,12$ and 15 \\
\hline RB per user (OFDMA, SC-FDMA) & $M_{R B}=1,2,3,4$ and 5 \\
\hline
\end{tabular}

\section{CM AND Link-Level Performance}

In this section, the $\mathrm{CM}$ and the link-level performance of MC-LDSMA is evaluated and compared with OFDMA and SC-FDMA.

\section{A. CM Comparison}

The problem of high CM is more critical for uplink due to the limited power at the user equipment. Low CM comparing to OFDMA was the major reason for adopting SC-FDMA as multiple access technique for 3GPP-LTE. So, it is crucial to evaluate the CM of MC-LDSMA and compare it with OFDMA and SC-FDMA. The complementary cumulative distribution function (CCDF) is used as a measure of the CM. The CCDF of $\mathrm{CM}$ denotes the probability that $\mathrm{CM}$ exceeds a certain value $C M_{0},\left(\operatorname{Pr}\left\{C M>C M_{0}\right\}\right)$. In addition to the CCDF curves, we will compare the $\mathrm{CM}$ values that are exceeded with probability less than $0.1 \%$, 99.9-percentile $\mathrm{CM}$, which is defined by $\operatorname{Pr}\left(C M>C M_{99.9}\right)=10^{-3}$ [9]. The CCDF of $\mathrm{CM}$ is calculated by Monte Carlo simulation. In practical systems, the user allocated subcarriers that grouped into basic units called Resource Blocks (RB) [10]. Considering that the number and the distribution of RBs allocated to the user affects the CM [11], we generated the results for different number of RBs per user. Also, all the possible distributions for the allocated RBs will be considered, however, for SC-FDMA only adjacent RB allocation is considered. The simulation parameters used for CM evaluation are listed in Table I. Considering that MC-LDSMA uses spreading, the allocated RBs per user will be more than OFDMA and SC-FDMA. The CM of OFDMA and MC-LDSMA is the same for different modulation orders, hence, only the results of 16QAM modulation is presented.

Figure 3 shows the CCDF curves of the CM and Table II lists the 99.9-percentile $\mathrm{CM}$ values that each signal experiences. It can be seen from the figure that MC-LDSMA has CM lower than OFDMA. MC-LDSMA has 99.9-percentile CM $1.15 \mathrm{~dB}$ less than OFDMA. On the other hand, comparing to SC-FDMA, MC-LDSMA has higher CM values especially for the QPSK modulation. For the worst case (QPSK modulation), MC-LDSMA has a 99.9-percentile CM $3 \mathrm{~dB}$ more than SCFDMA. Consequently, MC-LDSMA requires $3 \mathrm{~dB}$ more backoff to avoid the non-linear region of high-power amplifiers. This shows SC-FDMA outperforms MC-LDSMA in the CM. However, as we will show in the next section, this loss will be compensated by better link-level performance.

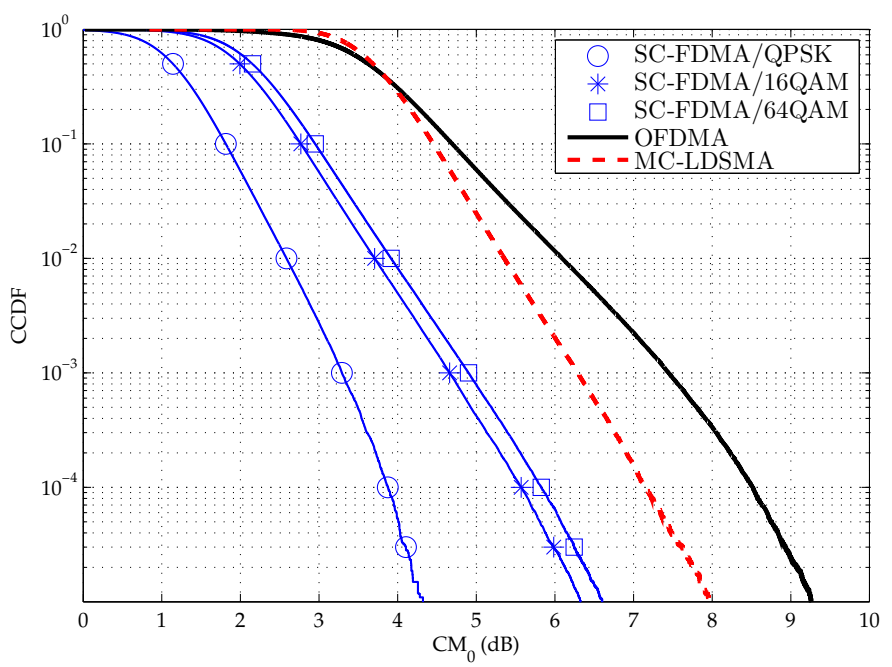

Fig. 3. CM comparison for SC-FDMA, OFDMA and MC-LDSMA.

TABLE II

Comparison of 99.9-PERCENTILE CM.

\begin{tabular}{|c||c|c|c|}
\hline & QPSK & 16QAM & 64QAM \\
\hline \hline SC-FDMA & 3.3 & 4.6 & 4.9 \\
\hline OFDMA & \multicolumn{3}{|c|}{7.45} \\
\hline MC-LDSMA & \multicolumn{3}{|c|}{6.3} \\
\hline Gain over SC-FDMA & -3 & -1.64 & -1.4 \\
\hline Gain over OFDMA & \multicolumn{3}{|c}{1.15} \\
\hline
\end{tabular}

TABLE III

LINK-LEVEL SiMULATION PARAMETERS

\begin{tabular}{|c|c|}
\hline \multicolumn{1}{|c|}{ Parameter } & Value \\
\hline \hline FFT size & 512 \\
\hline Subcarrier spacing & $15 \mathrm{KHz}$ \\
\hline Subcarriers per RB & 12 \\
\hline Number of data RB & $N_{R B}=25$ \\
\hline MC-LDSMA Scheme & $d_{v}=3, d_{c}=3$ \\
\hline RB per user (MC-LDSMA) & $M_{R B}=3,6,9,12$ and 15 \\
\hline RB per user (OFDMA, SC-FDMA) & $M_{R B}=1,2,3,4$ and 5 \\
\hline Number of users & $K=25,12,8,6$ and 5 \\
\hline Channel coding & Half-rate convolutional code \\
\hline
\end{tabular}

\section{B. Link-Level Comparison}

Here, we present the link-level performance comparison between the three multiple access techniques in terms of BLER. As shown earlier in the CM evaluation, the highest gain of SC-FDMA over MC-LDSMA is with QPSK modulation. Therefore, here we will focus on the BLER performance for QPSK modulation. The simulation parameters are listed in Table III. The aim is to show the gains achieved by frequency diversity due to the spreading used in the MC-LDSMA technique. Figures 4 and 5 show the BLER versus $E b / N_{0}$ (energy per bit to noise power spectral density ratio) for OFDMA, SCFDMA and MC-LDSMA. It can be observed from Fig. 4 that SC-FDMA and OFDMA have the same BLER performance. As only one RB is allocated to each user, the same frequency diversity is achieved by both systems. However, MC-LDSMA 


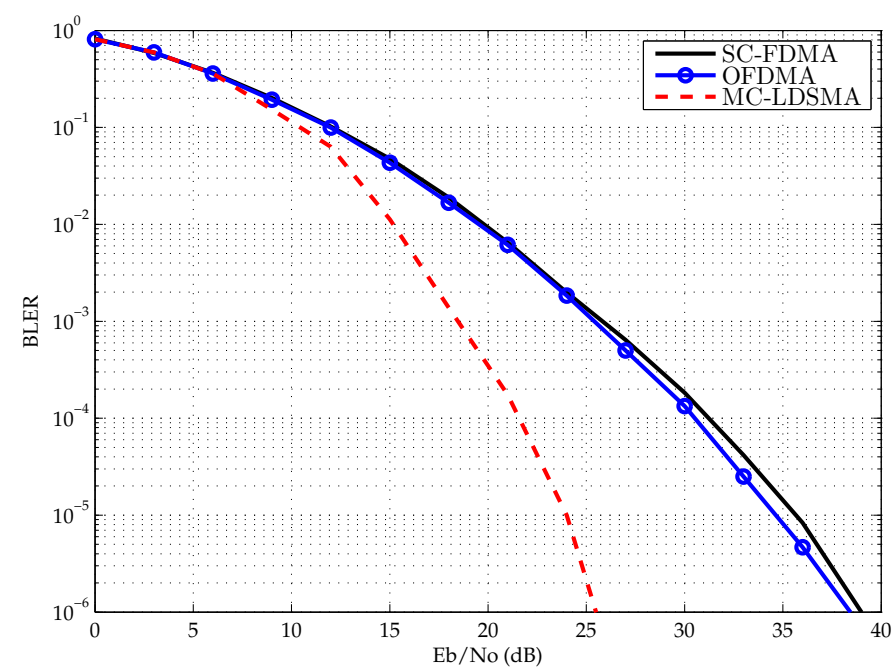

Fig. 4. BLER comparison for SC-FDMA, OFDMA (one RB per user) and MC-LDSMA (three RBs per user).

achieves better performance due to the frequency diversity gained by spreading on more than one resource block. On the other hand, in Fig. 5 we can see that OFDMA achieves better BLER comparing to SC-FDMA. This can be easily justified by taking into account that in SC-FDMA the RBs allocated to each user has to be adjacent [12], while in OFDMA the allocated RBs can be distributed. Consequently, the achieved frequency diversity is higher in OFDMA, which resulting in better link-level performance. MC-LDSMA maintains its superiority to OFDMA and SC-FDMA. For the Quality of Service (QoS) classes defined in 3GPP-LTE [13] there are three BLER thresholds; $10^{-2}, 10^{-3}$ and $10^{-6}$. Table IV lists the required $E b / N_{0}$ to achieve the BLER threshold averaged over all the simulated cases. As we can see from the results, MC-LDSMA achieves significant gains in the required $E b / N_{0}$ for the BLER thresholds, especially for low BLER thresholds. It is worth mention that the $10^{-2}$ BLER threshold is only required by the conversational service and all the other services require the other two BLER thresholds.

Considering the CM and link-level performance results, we can conclude that MC-LDSMA outperforms OFDMA in CM and link-level performance. On the other hand, comparing to SC-FDMA, MC-LDSMA has higher CM values, especially for QPSK modulation. Nevertheless, the loss due to the required back-off is compensated by better link-level performance making MC-LDSMA outperforms SC-FDMA. Table V lists the net gain, for QPSK modulation, achieved by MC-LDSMA over SC-FDMA and OFDMA considering the CM and linklevel performance. As it is clear from the table, MC-LDSMA achieves high gains over SC-FDMA and OFDMA. So, by using MC-LDSMA technique the overall required transmit power can be reduced comparing to SC-FDMA and OFDMA. This power saving will be reflected on the battery life of the user equipment and cell coverage can be increased.

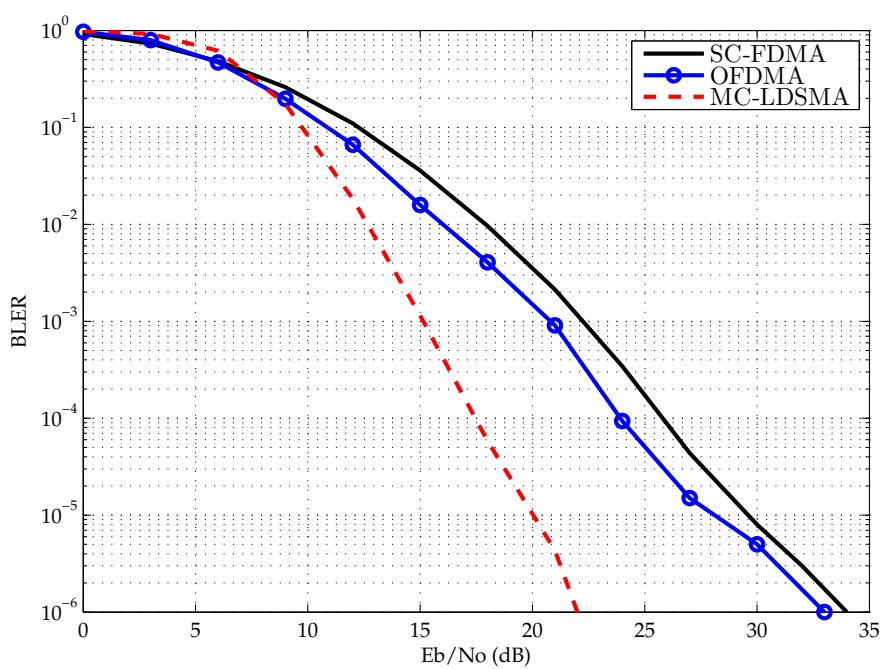

Fig. 5. BLER comparison for SC-FDMA, OFDMA (three RBs per user) and MC-LDSMA (nine RBs per user).

TABLE IV

REQuiRED $E b / N_{0}$ (DB) TO ACHIEVE THE BLER THRESHOLD.

\begin{tabular}{|c||c|c|c|}
\hline & $10^{-2}$ & $10^{-3}$ & $10^{-6}$ \\
\hline \hline SC-FDMA & 18.3 & 23.2 & 35.2 \\
\hline OFDMA & 17 & 21.7 & 34.6 \\
\hline MC-LDSMA & 13.5 & 16.2 & 23.2 \\
\hline Gain over SC-FDMA & 4.8 & 7 & 12 \\
\hline Gain over OFDMA & 3.5 & 5.5 & 11.4 \\
\hline
\end{tabular}

TABLE V

MC-LDSMA NET GAIN OVER SC-FDMA AND OFDMA.

\begin{tabular}{|c||c|c|c|}
\hline & $10^{-2}$ & $10^{-3}$ & $10^{-6}$ \\
\hline \hline Gain over SC-FDMA & 1.8 & 4 & 9 \\
\hline Gain over OFDMA & 4.65 & 6.65 & 12.55 \\
\hline
\end{tabular}

\section{System-LeVel Performance}

In multiuser systems, radio resource allocation plays a key role in optimizing the performance of multiuser systems by exploiting the frequency and multiuser diversity gains. Power and subcarriers should be allocated to the users in a way that trade-off between the spectral efficiency and fairness. In general, fairness is necessary when there is a limited resource that has to be simultaneously shared between many users. The fairness problem is prominent in the wireless communication systems where it is more spectral-efficient to allocate the resource to the users close to the base station, which is not fair to the users far from the base station. Consequently, more effort to serve the cell-edge users should be done. This can be achieved by assigning weights to prioritize the users, where users with bad channel conditions will be assigned higher weights to give them more priority in the allocation algorithm. So, the optimization problem for radio resource allocation can be formulated as weighted sum-rate maximization as follows;

$$
\max _{p_{k, n}, u_{k, n}} \sum_{k=1}^{K} w_{k} \sum_{n=1}^{N} u_{k, n} r_{k, n}\left(p_{k, n}\right),
$$


subject to:

$$
\sum_{n=1}^{N} p_{k, n} \leq P_{k},
$$

where $w_{k}$ is the weight associated with user $k$ and $r_{k, n}\left(p_{k, n}\right)$ is the rate of user $k$ on subcarrier $n . u_{k, n}$ is the subcarrier allocation index, where $u_{k, n}$ equal to 1 if subcarrier $n$ allocated to user $k$ and 0 otherwise. In addition to the power constraint (8), each multiple access technique has a specific constraint on the optimization problem in (7). For OFDMA and SC-FDMA, there is an exclusivity constraint on the subcarrier allocation where the subcarrier cannot be allocated for more than one user. The exclusivity constraint can be formulated as follows;

$$
\sum_{k=1}^{K} u_{k, n}=1 .
$$

This constraint brings down the system capacity as the subcarriers allocated to a user cannot be used by another user in the same time. Another constraint for SC-FDMA is that users can only be allocated subcarriers that are adjacent [12], which make it less efficient in utilizing the channel diversity. As been pointed out before, for MC-LDSMA, there is no exclusivity in the subcarrier allocation and up to $d_{c}$ users can share the same subcarrier. So, the exclusivity constraint in (9) can be replaced by more relaxed one for MC-LDSMA as follows;

$$
\sum_{k=1}^{K} u_{k, n} \leq d_{c}
$$

This subcarrier allocation flexibility in MC-LDSMA can significantly improve the system spectral efficiency by allowing the subcarrier to be reused by other users. By allowing more users to share the same subcarrier, the spectral efficiency can be increased. However, there is a limitation on the value of $d_{c}$ as the receiver complexity will be increased by increasing $d_{c}$. Therefore, it is essential to balance between the system spectral efficiency and receiver complexity to achieve the desired performance. In our system-level performance evaluation of MC-LDSMA, we will chose different values of $d_{c}$ to see the effect on the spectral efficiency.

In this section, the system-level performance of $\mathrm{MC}$ LDSMA is compared with OFDMA and SC-FDMA under dynamic resource block allocation. Spectral efficiency and Jain's fairness index are used as the performance evaluation metrics. Equal power allocation over the allocated resource blocks is used. The radio resource allocation algorithm we proposed in [14] will be used here. For OFDMA and SC-FDMA, the algorithms proposed in [15] and [12] will be used, respectively. We have considered a hexagonal cell with $1 \mathrm{~km}$ radius and assumed that users' locations are randomly generated and uniformly distributed over the cell. The maximum transmit power of each user is $23 \mathrm{dBm}$ and the system bandwidth is $5 \mathrm{MHz}$ consisting of 25 resource blocks. The link gain between the base station and a user is given as the product of path loss, shadowing and fast fading effects. ITU pedestrian B channel model [16] is adopted for generating fast fading

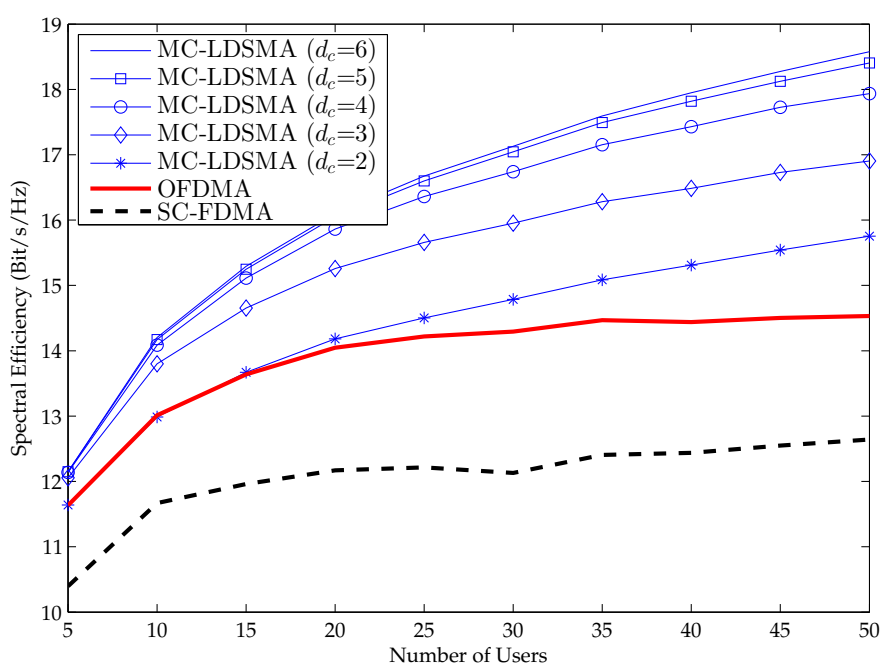

Fig. 6. Spectral Efficiency comparison for SC-FDMA, OFDMA and MCLDSMA.

and the simplified model [17] for the path loss. Lognormal shadowing is considered with mean value 0 and standard deviation of $8 \mathrm{~dB}$. The noise power spectral density is assumed to be $-173 \mathrm{dBm} / \mathrm{Hz}$. The users' weights are calculated as a function of the users' path losses to ensure fairness among the users by giving high priority to users far from the base station (cell-edge users). For MC-LDSMA system, the number of users per subcarrier $\left(d_{c}\right)$ is chosen to be between 2 and 6 .

Figure. 6 shows the spectral efficiency versus the total number of users for MC-LDSMA, OFDMA and SC-FDMA. As it can be observed from the figure, higher spectral efficiency is achieved by MC-LDSMA comparing to OFDMA and SCFDMA. Specifically, when the total number of users increased, OFDMA and SC-FDMA techniques become less competitive comparing to MC-LDSMA. The reason behind MC-LDSMA superiority is the non-exclusivity in the subcarrier allocation, which results in two advantages. First, the subcarriers that allocated to the cell-edge users to achieve fairness can be allocated to the users close to the base station, which results in the high spectral efficiency. The second advantage is that MC-LDSMA is capable of supporting users more than the number of available resource blocks $\left(N_{R B}\right)$ at the same time, which increase the sum of users' transmitted power. On the other hand, OFDMA and SC-FDMA cannot support more than $N_{R B}$ users simultaneously due to the allocation exclusivity. Also, the subcarriers used by the cell-edge users cannot be allocated to the users with good channel conditions.

In order to evaluate the fairness among the users, Fig. 7 shows Jain's fairness index for the three multiple access techniques, which is given by [18]:

$$
\text { Jain's fairness index }=\frac{\left(\sum_{k=1}^{K} R_{k}\right)^{2}}{K \sum_{k=1}^{K} R_{k}^{2}} .
$$

As the figure shows, MC-LDSMA is much fair comparing to OFDMA and SC-FDMA over all the simulated number of users $(K)$. This is due to the fact that cell-edge users 


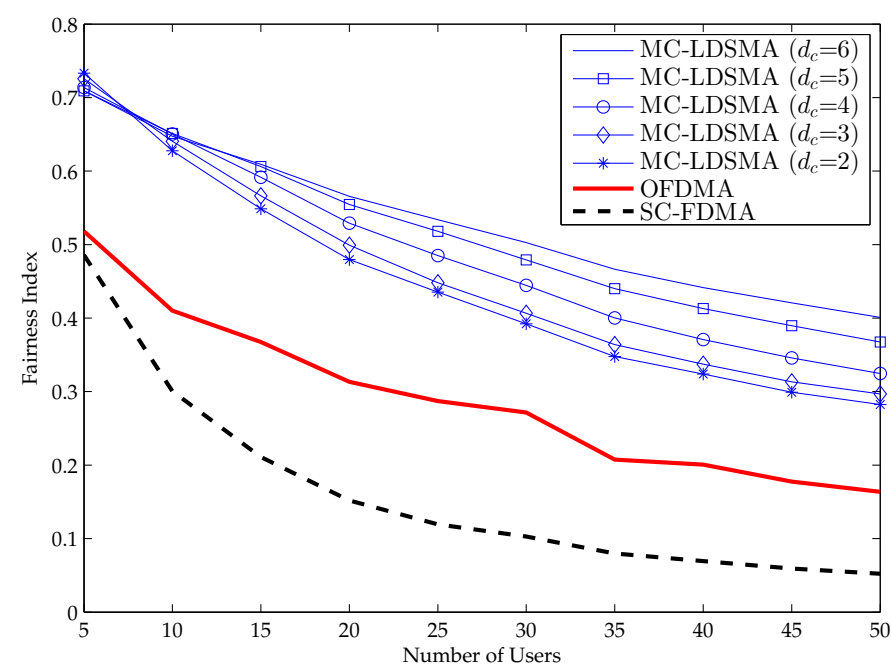

Fig. 7. Jain's fairness index comparison for SC-FDMA, OFDMA and MCLDSMA.

can achieve more rates with MC-LDSMA technique. Even for a small number of users, where the spectral efficiency gain is low, the gain of using MC-LDSMA is evident from the fairness performance evaluation. As the results show, for MC-LDSMA, when the number of users per subcarrier $\left(d_{c}\right)$ is increased more spectral efficiency and fairness can be achieved. However, at high values of $d_{c}$ the improvement in the system performance is marginal with the increase of $d_{c}$, especially the spectral efficiency. For example, as Fig. 6 shows, $d_{c}=5$ achieves almost the same spectral efficiency as $d_{c}=6$. This suggest that high values of $d_{c}$ are not necessary, and the receiver complexity can be kept affordable. In fact, the number of users per subcarrier can be adjusted to trade-off between system-level performance and receiver complexity. These improvements in the system-level performance can be translated into an increase in the number of supported users in the cell and users' data rates and cell coverage.

Considering the results altogether, the following main conclusions can be derived: MC-LDSMA achieves superior performance comparing to OFDMA and SC-FDMA in linklevel and system-level performance. MC-LDSMA requires less transmission power to achieve the BLER threshold comparing to SC-FDMA and OFDMA. Higher spectral efficiency and fairness can be achieved with MC-LDSMA. These gains can increase the battery life of the user equipment, cell coverage, number of supported users in the cell and users' data rates.

\section{CONCLusion}

Next generation mobile communication systems demands high spectral-efficient multiple access techniques. In this paper, we have investigated the expected gains of using MCLDSMA as a multiple access technique for next generation mobile communications. We evaluated the performance of MC-LDSMA and compared it with SC-FDMA and OFDMA using CM, link-level and system-level performance metrics through extensive Monte Carlo simulations. The numerical results showed the superiority of MC-LDSMA performance over SC-FDMA and OFDMA. It is shown that using MCLDSMA can reduce the required transmission power and increase the system spectral efficiency and fairness. Consequently, significant improvements can be achieved in terms of the battery life of the user equipment, cell coverage, number of supported users in the cell and users' data rates. Owning these advantages, MC-LDSMA represents a strong candidate for next generation mobile communication systems as a multiple access technique.

\section{ACKNOWLEDGMENT}

This work was supported by Huawei Technologies Co., Ltd, China.

\section{REFERENCES}

[1] J. Bingham, "Multicarrier modulation for data transmission: An idea whose time has come," IEEE Communications Magazine, vol. 28, no. 5, pp. 5-14, May 1990.

[2] Third Generation Partnership Project, Technical Specification Group Radio Access Network, "Physical layer aspects for evolved Universal Terrestrial Radio Access (UTRA)", 3GPP Std. TR 25.814 v. 7.0.0, 2006.

[3] IEEE Std. 802.16-2009, "Standard for Local and metropolitan area networks. Part 16: Air Interface for Broadband Wireless Access Systems", May 2009.

[4] H. G. Myung, J. Lim, and D. J. Goodman, "Single carrier FDMA for uplink wireless transmission," IEEE Vehicular Technology Magazine, vol. 1, no. 3, pp. 30-38, Sept. 2006.

[5] A. Montanari and D. Tse, "Analysis of belief propagation for non-linear problems: The example of CDMA (or: How to prove tanaka's formula)," in IEEE Information Theory Workshop, March 2006, pp. 160-164.

[6] 3GPP TSG RAN WG1 and 3GPP TSG RAN WG4, TDocs R4-040367, R1-040522 and R1-040642, "Comparison of PAR and Cubic Metric for power de-rating", May 2004.

[7] A. Behravan and T. Eriksson, "Some statistical properties of multicarrier signals and related measures," in IEEE Vehicular Technology Conference, vol. 4, May 2006, pp. 1854-1858.

[8] R. Hoshyar, F. Wathan, and R. Tafazolli, "Novel low-density signature for synchronous CDMA systems over AWGN channel," IEEE Transactions on Signal Processing, vol. 56, no. 4, pp. 1616-1626, April 2008.

[9] H. Myung, J. Lim, and D. Goodman, "Peak-to-average power ratio of single carrier FDMA signals with pulse shaping," in IEEE International Symposium on Personal, Indoor and Mobile Radio Communications, Sept. 2006, pp. 1-5.

[10] E. Dahlman, S. Parkvall, J. Sköld, and P. Beming, $3 G$ Evolution: HSPA and LTE for Mobile Broadband. Academic Press, 2007.

[11] G. Huang, A. Nix, and S. Armour, "Impact of radio resource allocation and pulse shaping on PAPR of SC-FDMA signals," in IEEE International Symposium on Personal, Indoor and Mobile Radio Communications, Sept. 2007, pp. 1-5.

[12] I. Wong, O. Oteri, and W. Mccoy, "Optimal resource allocation in uplink SC-FDMA systems," IEEE Transactions on Wireless Communications, vol. 8, no. 5, pp. 2161-2165, May 2009.

[13] 3GPP, Technical Specification Group Services and System Aspects, "Policy and charging control architecture", TS 23.203 V9.9.0, June 2011.

[14] M. Al-Imari, M. Imran, R. Tafazolli, and D. Chen, "Subcarrier and power allocation for LDS-OFDM system," in IEEE Vehicular Technology Conference, May 2011, pp. 1-5.

[15] C. Y. Ng and C. W. Sung, "Low complexity subcarrier and power allocation for utility maximization in uplink OFDMA systems," IEEE Transactions on Wireless Communications, vol. 7, no. 5, pp. 1667-1675, May 2008.

[16] ITU, Guidelines for Evaluation of Radio Transmission Technologies for IMT-2000. ITU-R Recommendations on IMT, M.1225, 1997.

[17] A. Goldsmith, Wireless Communications. Cambridge University Press, 2005.

[18] R. K. Jain, D.-M. W. Chiu, and W. R. Hawe, "A quantitative measure of fairness and discrimination for resource allocation in shared computer systems," DEC Technical Report 301, Sept. 1984. 